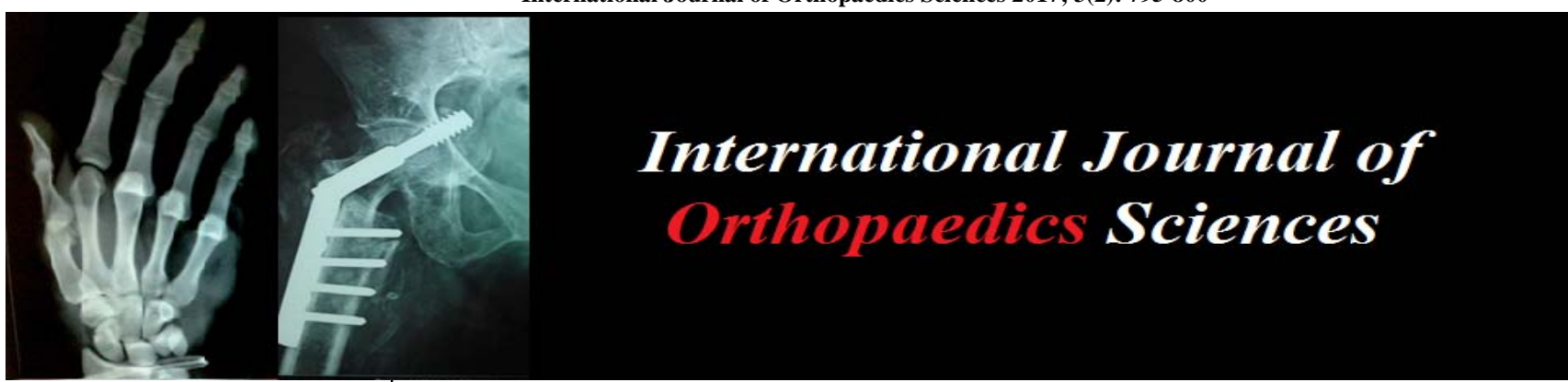

ISSN: $2395-1958$

IJOS 2017; 3(2): 795-800

(C) 2017 IJOS

www.orthopaper.com

Received: 25-02-2017

Accepted: 26-03-2017

Dr. Sachin NS

Associate Professor in

Department of Orthopaedics in SSIMS \& RC, Davangere,

Karnataka, India

Dr. Praveen M Anvekar

Associate Professor in

Department of Orthopaedics in

SSIMS \& RC, Davangere,

Karnataka, India

Dr. M Venkataramana Rao Professor in Department of Orthopaedics in SSIMS \& RC, Davangere, Karnataka, India

Dr. J Manjunath

Professor \& HOD Department of Orthopaedics in SSIMS \& RC,

Davangere, Karnataka, India

Dr. Kalwa Dinesh

Post Graduate in Department of Orthopaedics in SSIMS \& RC, Davangere, Karnataka, India

Correspondence

Dr. Sachin NS

Associate Professor in

Department of Orthopaedics in

SSIMS \& RC, Davangere,

Karnataka, India

\section{Clinical and functional outcome following total knee arthroplasty in primary osteoarthritis knees using PCL substituting prosthesis}

\author{
Dr. Sachin NS, Dr. Praveen M Anvekar, Dr. M Venkataramana Rao, \\ Dr. J Manjunath and Dr. Kalwa Dinesh
}

DOI: http://dx.doi.org/10.22271/ortho.2017.v3.i2i.85

\section{Abstract}

Background: Total knee replacement surgery is considered as a treatment of choice in patients with advanced arthritis, especially in patients where conservative treatment have been failed. Total knee replacement is considered as second most successful surgery in Orthopaedics, which gives good pain relief, function and disabilities following advanced arthritis.

Materials \& methods: 60 primary total knee replacement surgeries in 50 patients done using posterior stabilized (PS) Depuy's allpoly fixed bearing tibial cemented components, who were operated between March 2015 to August 2015, were prospectively enrolled into our study. This study was conducted at SS Institute of Medical Sciences \& Research Centre, Davanagere, Karnataka. They were followed up for a minimum period of 1 year, and evaluated using knee society score. There were 24 right knees, 16 left knees, 10 bilateral knees.

Results: There was a significant improvement in function of the patients and we have achieved excellent results in 42 patients and good results in 8 patients, no patient had a poor result. At one year follow up an average pre-op Knee Clinical Score of 28.28 improved to an average postop Knee Clinical Score of 94.23 and an average pre-op Knee Functional Score of 41.67 improved to an average post-op Knee Functional Score of 87.33.There was no case of infection or radiolucency's at the end of one year.

Conclusion: Among 50 patients who were diagnosed with primary osteoarthritis of knee, pain and deformity were the main presenting complaint. Inability to flex the affected knee and difficulty in doing activities like squatting and sitting cross legged was seen in most of the patients. In Indian scenario where patient comes late with gross varus deformity and fixed flexion deformity, it is difficult to balance the knee with retention of posterior cruciate ligament, where as Posterior Cruciate ligament Substituting Total Knee Replacement gives reproducible and good results, hence it is the preferred mode of management.

Keywords: Osteoarthritis, posterior stabilized knee, knee functional score

\section{Introduction}

In most arthritic knees, some degree of instability, deformity, contracture or a combination of these elements, can be found ${ }^{[1,2,3]}$. The common causes of arthritis of the knee include Osteoarthritis (OA), Rheumatoid Arthritis (RA), Juvenile Rheumatoid Arthritis, PostTraumatic Arthritis or Secondary Osteoarthritis and other types of inflammatory arthritis.

Osteoarthritis is thought to be the most prevalent chronic joint disease. The incidence of osteoarthritis is rising because of the ageing population and the epidemic of obesity. Pain and loss of function are the main clinical features that lead to treatment, including nonpharmacological, pharmacological, and surgical approaches ${ }^{[4]}$.

The concept of improving knee joint function by modifying the articular surfaces has received attention since the 19th century. The surgical techniques have varied from soft tissue interposition arthroplasty to resection arthroplasty to surface replacement arthroplasty. In surface replacement arthroplasty different types of prosthesis were developed to address the complex knee kinematics.

Total Knee Arthroplasty (TKA) is now a reliable treatment for severe arthritis. Various systems are available with specific features regarding the geometry of the components, 
the degree of conformity of the articulating surface and the anchoring technique. With the advent of these varied types of prosthesis it became necessary to conduct studies for assessing the outcome of different prosthesis. Hence different scoring systems were devised for assessing the outcome of total knee replacement.

The Knee Society Score System is subdivided into a knee score that rates only the knee joint itself and a functional score that rates the patient's ability to walk and climb stairs. The dual rating system eliminates the problem of declining knee scores associated with patient infirmity ${ }^{[5]}$.

\section{Materials and methods}

A study, was carried out at SS Institute of Medical Sciences \& Research Centre, Davanagere, Karnataka, where 50 patients were operated with 60 Total knee replacement surgeries, between March 2015 to August 2015.

They were followed up for a minimum period of 1 year by the senior author and evaluated for clinical outcome using XRAYS, and Knee society score.

A written consent for participation in this prospective study was obtained from all patients.

Patients were followed at 1month, 3 months, 6 months and 1 year, post surgery.

The pre and post-operative Knee Clinical Score and Knee Functional Score was compared using Paired t-test and for correlation Pearson correlation co-efficient formula was used.

\section{Inclusion criteria}

- Patients with primary osteoarthritis who underwent Total Knee Arthroplasty who gave written informed consent.

- Both bilateral and unilateral primary osteoarthritis patients.

\section{Exclusion criteria}

- Patients having gross ligamentous imbalance and requiring constrained implants.

- Chronic pain unrelated to knee OA

- Severe obesity negatively influenced postoperative knee society scores.

- Intraoperative and Postoperative fractures. (Periprosthetic fractures)

\section{Pre operative evaluation}

Detailed history of all patients was taken. All patients were assessed clinically and functionally using the Knee Society Score ${ }^{5}$. The preoperative medical evaluations of all patients were done to evaluate any potential complications that can be life-threatening or limb-threatening. Out of 50 patients, 23 patients had only varus deformity and 27 patients had both varus and fixed flexion deformity.

\section{Radiographic assessment}

- Standard guidelines were utilized to obtain knee radiographs - standing anteroposterior view, a lateral view and a skyline view of the patella.

- Any collateral ligament laxity, subluxation of tibia, presence of osteophytes, any bone defects in the tibia and femur and the quality of bone was assessed.

- Sizing of the femoral and tibial components was also done.

\section{Operative technique}

All patients after thorough pre-op evaluation were taken up for surgery under combined epidural and spinal anesthesia, all patients received $1 \mathrm{grm}$ of ceftriaxone, and $1 \mathrm{grm}$ of tranexamic acid 15 minutes prior to surgery and tourniquet inflation. A standard medial parapatellar approach was used with knee in 90 degrees flexion. Distal femoral 5-7 degrees of valgus cut is made with help of intrameduallary jig and tibial cut is done perpendicular to mechanical axis with the help of extrameduallary jig. Femoral and tibial sizing done and appropriate preparation is made. Soft tissue balancing done with trial components insitu, in both flexion and extension. In all patients we have used depuy femoral component on femoral side, and on tibial side posterior stabilized allpoly tibial component. Patella is not resurfaced and circumferential denervation of the patella was done. At the end of procedure tourniquet is removed haemostasis is achieved and wound closed in layers over drain.

\section{Results}

All patients are mobilised from post-op day 1. Drain was removed on $2^{\text {nd }}$ post-op day following which intensive physiotherapy under supervision was given to all patients.

Unilateral operated patients are discharged after 1 week, bilateral patients are discharged after 2 weeks. Patients were evaluated clinically and radiologically at an interval of 1month, 3 months, 6 months and 1 year, in Orthopaedics OPD.

- This is a consecutive study of 50 patients who underwent Total Knee Arthroplasty using posterior cruciate substituting design.

- The majority of the patients were from the age group of 61-65 years which accounts for $36.7 \%$ of patients in our study. The youngest patient was 52 years of age and the oldest patient was 79 years. The mean age was 62.07 .

- There was a female predominance in the ratio of 3:2 in our study, accounting for $60 \%$ of the patients.

- There was a predominance of right side accounting for 36.67 of the patients.

- The diagnosis was Primary Osteoarthritis of knee in all the cases.

\section{Knee clinical score}

\begin{tabular}{|c|c|c|c|c|c|c|c|}
\hline & N & Mean & Median & Mode & Standard deviation & Minimum & Maximum \\
\hline PRE-OP & 50 & 28.28 & 27.50 & 26 & 7.42 & 14 & 40 \\
\hline POST-OP & 50 & 94.23 & 96.00 & 98 & 5.41 & 80 & 99 \\
\hline
\end{tabular}

The mean pre - op Knee Clinical Score was 28.28 in this study which improved to a mean post - op score of 94.23

\section{Knee functional score}

\begin{tabular}{|c|c|c|c|c|c|c|c|}
\hline & N & Mean & Median & Mode & Standard deviatiom & Minimum & Maximum \\
\hline PRE-OP & 50 & 41.67 & 45.00 & 45 & 8.48 & 20 & 50 \\
\hline POST-OP & 50 & 87.33 & 90.00 & 90 & 6.86 & 60 & 90 \\
\hline
\end{tabular}


The mean pre - op Knee Functional Score was 41.67 in this study which improved to an mean post - op score of 87.33

\section{Knee clinical score and knee functional score cross- tabulation}

\begin{tabular}{|c|c|c|c|c|c|}
\hline & \multicolumn{5}{|c|}{ Knee functional score } \\
\hline Knee clinical score & & Excellent & Good & Fair & Total \\
\hline Excellent & & 40 & 02 & 00 & 42 \\
\hline Good & & 00 & 06 & 02 & 08 \\
\hline Poor & 00 & 00 & 00 & 00 & 00 \\
\hline
\end{tabular}

- According to the Knee Society Functional Scoring system of the 50 patients assessed in this study 42 patients had Excellent, 06 patients had Good and 2 patients had Fair results.
Comparison between pre-op and post-op knee clinical and functional scores

\begin{tabular}{|c|c|c|c|}
\hline & \multicolumn{2}{|c|}{ Paired difference } & \multirow{2}{*}{ P-value } \\
\cline { 2 - 3 } & Mean & Standard deviation & \\
\hline Pre op kcs-post op kcs & 65.95 & 7.293 & $<0.001$ \\
\hline Pre op kfs-post op kfs & 45.67 & 8.410 & $<0.001$ \\
\hline
\end{tabular}

- The difference between the means of pre - op KCS and post - op KCS was 65.95 (62.65 to $69.25,95 \% \mathrm{CI})$. The $\mathrm{P}$ value was significant $(<0.001)$ when the pre - op and post - op Knee Clinical Scores were compared.

- The difference between the means of pre - op KFS and post - op KFS was 45.67 (43.39 to $47.95,95 \% \mathrm{CI})$. The $\mathrm{P}$ value was significant $(<0.001)$ when the pre - op and post - op Knee Functional Scores were compared.
The $\mathrm{P}$ value was highly significant $(<0.00001)$, indicating significant co-relation between Clinical Knee Score and Functional Knee Score at one year follow up of Posterior Cruciate Sacrificing Total Knee Arthroplasty as the result is significant at the value of $\mathrm{p}<0.05$.
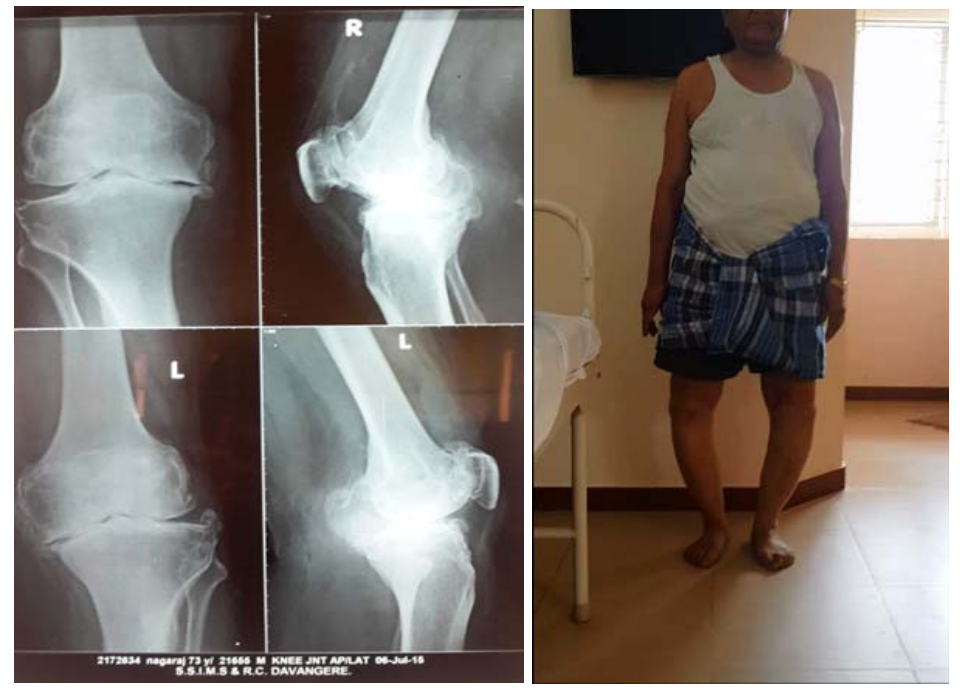

Pre-op images
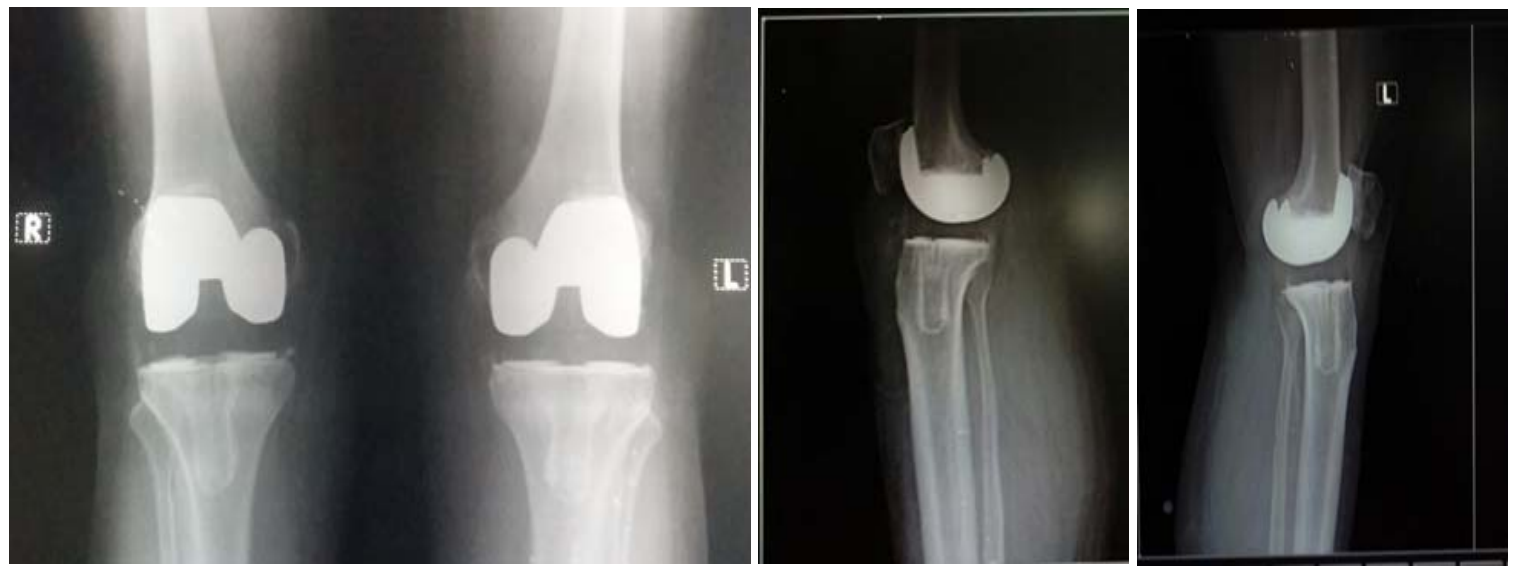

1 year Post-op x-rays

$\sim 797 \sim$ 


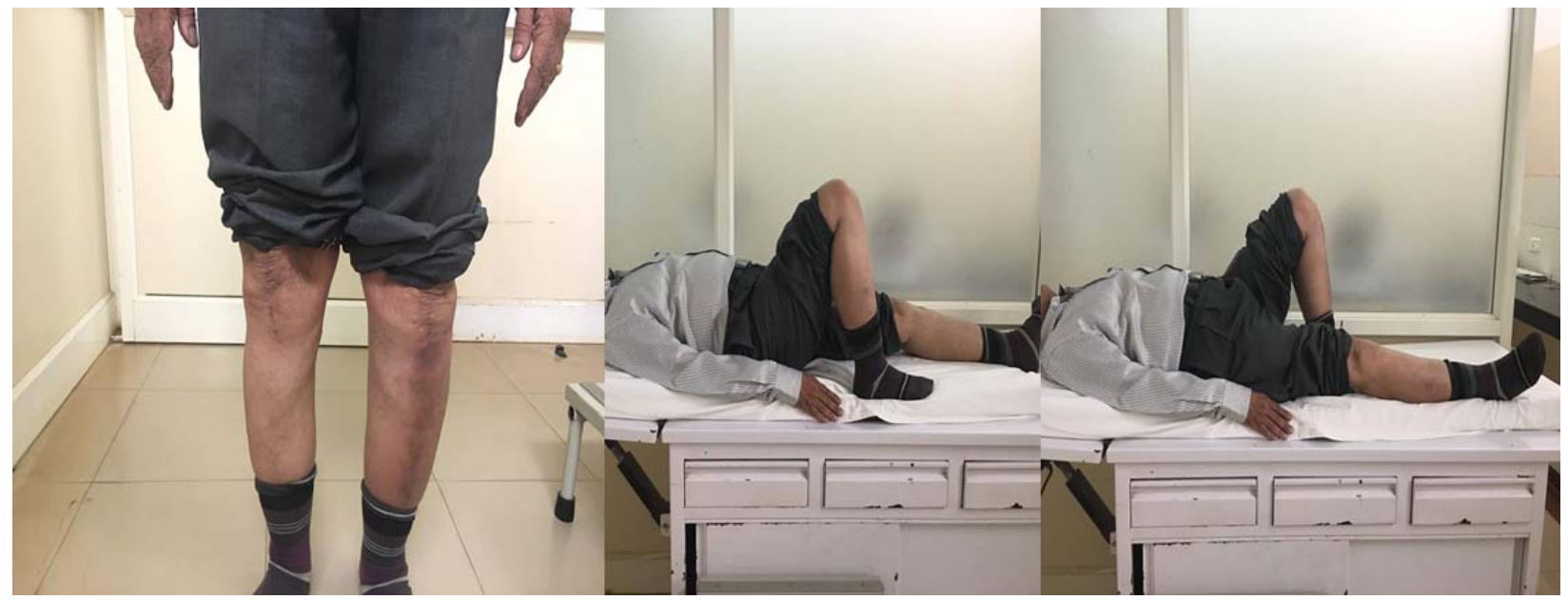

1 year follow-up clinical images

There was no change in radiological outcome assessment in one year follow up.

\section{Discussion}

Total Knee Arthroplasty is generally an effective procedure and is associated with substantial functional improvement. Elderly patients who were having difficulty mobilizing because of degenerative arthritis found good relief after Total Knee Arthroplasty. There was a substantial relief of joint pain, increased mobility, correction of deformity and an improvement in the quality of life of the patients following Posterior Cruciate Sacrificing Total Knee Arthroplasty. With the varied amount of implant designs available the posterior cruciate substituting design was found to be effective ${ }^{[6]}$.

C. Buz Swanik found that following total knee arthroplasty, patients were able to reproduce joint position and improve mobility significantly. These changes may result from the retensioned capsulo-ligamentous structures and reduced pain and inflammation. The balance index also improved significantly from the preoperative to the postoperative evaluation. The group treated with the posterior stabilized prosthesis more accurately reproduced joint position when the knee was extended from a flexed position. Retention of the posterior cruciate ligament does not appear to significantly improve proprioception and balance compared with those functions in patients with a posterior stabilized total knee design ${ }^{[7]}$.

Gait analysis by Andriacchi and Galante, Kelman et al., and others found that individuals with PCL-retaining prostheses have a more symmetrical gait, especially during stair climbing, than do individuals with either PCLsacrificing/PCLsubstituting designs. They showed decreased knee flexion during stair climbing and a tendency to lean forward in a quadriceps-sparing posture in patients with PCLsacrificing/PCL-substituting designs. They postulated that these observations may indicate inadequate rollback of these designs or possibly the loss of a proprioceptive role of the PCL. These observations have been cited as reasons to retain the PCL ${ }^{[8]}$.

Gait analysis by Wilson et al. contradicts the conclusions of these earlier studies, however, after comparing PCLsubstituting/sacrificing knees with normal controls. These earlier observations are refuted further by in vivo studies by Stiehl et al.; Victor, Banks, and Bellemans and Dennis et al., who used fluoroscopy during single-stance deep knee bends to show a paradoxical forward translation of the femorotibial contact point during weight bearing flexion in some PCLretaining knees; PCL-substituting/sacrificing knees studied showed more uniform femoral rollback ${ }^{[9,10]}$.

Whether the PCL is salvaged or sacrificed at TKR has been much debated ${ }^{[11]}$. Posterior glide and roll of the femorotibial contact region with flexion is influenced by the PCL. Isolated removal of the PCL increases the flexion gap. While it may be attractive in gaining surgical access and subsequent flexion, PCL sacrifice encourages posterior tibial subluxation. A tibial post and femoral cam, or a dished polyethylene insert with raised anterior lip, may resurrect both stability and femorotibial rollback. Evidence suggests that using PCLsubstituting/sacrificing designs increases post-operative knee flexion. This could perhaps be because of more normal kinematics. Fluoroscopic studies demonstrate increased femoral rollback using the cam-post articulation as compared with some PCL-retaining systems ${ }^{[12]}$.

Many authors argue that the PCL is diseased with various forms of arthritis and contracture and is difficult to balance reproducibly. Although intraoperative tests of PCL balance have been devised by advocates of PCL retention, such as Ritter and Scott, other investigators, including Maloney et al., have stated that it is difficult, even in a laboratory setting, to reproduce near-normal PCL strain and function in a PCLretaining knee arthroplasty $[13,14,15]$.

A randomized trial by Maruyama et al ${ }^{[16]}$, comparing patients with bilateral TKR, identified a greater range of movement with PCL substituting/sacrificing as compared with PCLretaining TKR. Clark et al ${ }^{[17]}$ noted increased flexion with PCL substitution at two years after surgery. A recent metaanalysis ${ }^{[18]}$ concluded that there was an improvement in flexion of $8^{\circ}$ in PCL-substituted designs compared with PCL retention. However, any improvement in flexion seemed limited and not associated with improved function. Other investigators have not detected improved flexion with PCL substitution/sacrificing $[19,20,21]$. The PCL may not function even when a PCL-retaining design is used ${ }^{[22]}$.

A Cochrane review [23] indicated no difference in clinical outcome when the PCL was retained or resected.

In this study 50 patients who met the inclusion criteria, all the knees were operated using a Posterior Cruciate Sacrificing design.

Robert L Barrack et al. found that total knee arthroplasty with retention of the patella yielded clinical results that were comparable with those after total knee arthroplasty with patellar resurfacing ${ }^{[24]}$. 
Robert L Barrack et al. concluded that postoperative anterior knee pain is related either to the Component design or to the details of the surgical technique, such as component rotation, rather than to whether or not the patella is resurfaced ${ }^{[25]}$.

Nutton concluded that knee function was not improved by patella resurfacing when compared to a matched group of patients without resurfacing ${ }^{[26]}$.

Wood et al concluded that total knee arthroplasty with patellar resurfacing exhibited inferior clinical results as compared to total knee arthroplasty with patellar retention. Total knee arthroplasty with patellar resurfacing exhibited significant limitation of knee extension, which was significantly associated with the presence of post-surgery anterior knee pain ${ }^{[27]}$.

Patellar resurfacing does not guarantee a painless patellofemoral joint ${ }^{[28,29]}$.

The KAT failed to demonstrate significant benefit from patellar resurfacing ${ }^{[30]}$.

In our study, all of the patellas were resurfaced. All patellas were circumferentially denervated. There was no anterior knee pain in any of our subjects.

The Knee Society Score was used to assess the outcome of Total knee Arthroplasty. The knee Society Score rating system was a logical outgrowth of the Hospital for Special Surgery (HSS) rating system.

The Knee Society Score system separates findings in the operated knee with findings in the patients function. The system is subdivided into a knee clinical score that rates only the knee joint itself and a knee functional score that rates the patient's ability to walk and climb stairs. The scoring system combines a relatively objective knee clinical score that is based on the clinical parameters and a knee functional score based on how the patients perceives that knee function with specific activities.

In our study there was significant improvement of Knee Clinical Score and Knee Functional Score following Posterior Cruciate Sacrificing Total Knee Arthroplasty. The Knee Society Roentgenographic evaluation and scoring system was developed for uniform reporting of roentgenographic results of Total Knee Arthroplasty. In our minimum period of one year follow up study the component position and knee alignment was well maintained. Long term follow up results are needed to strengthen the study.

\section{Conclusion}

Total Knee Arthroplasty improves the functional ability of the patient and the ability of the patient to get back to pre-disease state, which is to have a pain free mobile joint, as reflected by the improvement in the post-op Knee Clinical Score and Knee Functional Score.

In an Indian scenario where patient comes late with gross varus deformity and fixed flexion deformity, Posterior Cruciate Sacrificing Total Knee Replacement will give greater mobility in flexion especially in fixed flexion deformity, so it can be preferred mode of management.

With the use of Posterior Cruciate Sacrificing Design, at one year follow up an average pre-op Knee Clinical Score of 28.28 improved to an average postop Knee Clinical Score of 94.23 and an average pre-op Knee Functional Score of 41.67 improved to an average post-op Knee Functional Score of 87.33.

There was significant correlation between the Knee Clinical Score and Knee Functional Score at one year follow up.

The Knee Society Score is an effective scoring system as it incorporates clinical and functional outcome following Total
Knee Arthroplasty.

\section{References}

1. Vail TP, Lang JE. Insall and Scott surgery of the knee. 4th ed. Philadelphia: Churchill Livingstone, Elsevier, 2006, 1455-1521.

2. Insall J, Ranawat CS, Scott WN, Walker P. Total condylar knee replacement. Preliminary report. Clin Orthop Relat Res. 1976; 120:149-54.

3. Kim RH, Scott WN. Operative techniques: total knee replacement. Philadelphia: Saunders-Elsevier. 2009, 91103.

4. Bijlsma JW, Berenbaum F, Lafeber FP. Osteoarthritis: an update with relevance for clinical practice. Lancet. 2011; 18;377:2115-26.

5. John Insall N, Lawrence Dorr D, Richard Scott D, Norman Scott W. Rationale of The Knee Society Clinical Rating System. Clin Orthop. 1989; 248:13-14.

6. Dennis DA, Komistek RD, Stiehl JB. Range of motion after total knee arthroplasty: the effect of implant design and weight-bearing conditions. J Arthroplasty. 1998; 13:748.

7. Buz Swanik C. Proprioception, kinesthesia, and balance after total knee arthroplasty with cruciate retaining and posterior stabilized prostheses, J Bone Joint Surg. 2004; 86:328-34.

8. Andriacchi TP, Galante JO, Fermier RW. The influence of total knee replacement design on walking and stair climbing. J Bone Joint Surg. 1982; 64:1328.

9. Dennis DA, Clayton ML, O'Donnell S. Posterior cruciate condylar total knee arthroplasty: average 11-year followup examination. Clin Orthop Relat Res. 1992; 281:168.

10. Dennis DA, Komistek RD, Stiehl JB. Range of motion after total knee arthroplasty: the effect of implant design and weight-bearing conditions. J Arthroplasty. 1998; 13:748.

11. Insall JN, Clarke HD. Historic development classification and characteristics of knee prostheses. New York: Churchill-Livingstone. 2001, 1516-53.

12. Dennis DA, Komistek RD, Colwell Jr CE. In vivo anteroposterior femorotibial translation of total knee arthroplasty: a multicenter analysis. Clin Orthop Relat Res. 1998; 356:47.

13. Ritter MA, Campbell E, Faris P. Long-term survival analysis of the posterior cruciate condylar total knee arthroplasty: a 10-year evaluation. J Arthroplasty. 1989; 4:293.

14. Ritter MA, Harty LD, Davis KE. Simultaneous bilateral, staged bilateral, and unilateral total knee arthroplasty: a survival analysis. J Bone Joint Surg. 2003; 85:1532.

15. Maloney WJ, Schurman DJ. The effects of implant design on range of motion after total knee arthroplasty: total condylar versus posterior stabilized total condylar designs. Clin Orthop Relat Res. 1992; 278:147.

16. Maruyama S, Yoshiya S, Matsui N, Kuroda R, Kurosaka M. Functional comparison of posterior cruciate-retaining versus posterior stabilized total knee arthroplasty. J Arthroplasty. 2004; 19:349-53.

17. Clark CR, Rorabeck CH, MacDonald S, MacDonald D, Swafford J, Cleland D. Posterior-stabilised and cruciateretaining total knee replacement - A randomized study. Clin Orthop. 2001; 392:208-12.

18. Jacobs WCH, Clement D, Wymenga AB. Retention versus removal of the posterior cruciate ligament in total knee replacement. A systematic literature review within 
the Cochrane framework. Acta Orthopaedica. 2005; 76:757-68.

19. Shoji H, Wolf A, Packard S, Yoshino S. Cruciate retained and excised total knee arthroplasty. A comparative study in patients with bilateral total knee arthroplasty. Clin Orthop. 1994; 305:218-22.

20. Misra AN, Hussain MRA, Newton G. The role of the posterior cruciate ligament in total knee replacement. J Bone Joint Surg [Br]. 2003; 85-B:389-392.

21. Straw R, Kulkarni S, Attfield S, Wilton TJ. Posterior cruciate ligament at total knee replacement. Essential, beneficial or hindrance? J Bone Joint Surg [Br]. 2003; 85- B:671-74.

22. Dennis DA, Komistek RD, Colwell Jr CE. In vivo anteroposterior femorotibial translation of total knee arthroplasty: a multicenter analysis. Clin Orthop Relat Res. 1998; 356:47.

23. Bassett RW. Results of 1, 000 Performance knees: cementless versus cemented fixation. J Arthroplasty. 1998; 13:409-13.

24. Robert Barrack L. Resurfacing of the patella in total knee arthroplasty: a prospective, randomized, double-blind study. J Bone Joint Surg. 1997; 79:1121-31.

25. Robert Barrack L. Patellar resurfacing in total knee arthroplasty. J Bone Joint Surg. 2001; 83:1376-81.

26. Nutton. The functional outcome following total knee replacement with or without patella resurfacing. British Association for Surgery of the Knee. 2001, 27-28.

27. Wood. Clinical outcomes and walking analysis after total knee arthroplasty with and without patellar resurfacing: a prospective randomized trial. J Bone Joint Surg. 2005, 338-39.

28. Pakos EE, Ntzani EE, Trikalinos TA. Patella resurfacing in total knee arthroplasty: a meta-analysis. J Bone Joint Surg [Am]. 2005; 87-A:1438-45.

29. Wood DJ, Smith AJ, Collopy D, White B, Brankov B, Bulsara MK. Patella resurfacing in total knee arthroplasty: a prospective randomised trial. J Bone Joint Surg [Am]. 2002; 84-A:187-193.

30. Trial Group KAT. The Knee Arthroplasty Trial (KAT) Design features, baseline characteristics and two-year functional outcomes after alternative approaches to knee replacement. J Bone Joint Surg [Am]. 2009; 91:134-141. 